\title{
Traduire
}

Une autre perspective sur r tr traduction

Revue française de la traduction

$235 \mid 2016$

Luxe, mode... et traduction !

\section{Traduire la mode : un art à part entière}

\section{Percy Balemans}

Traducteur : Nelia Fahloun

\section{CpenEdition}

\section{Journals}

Édition électronique

URL : https://journals.openedition.org/traduire/840

DOI : 10.4000/traduire.840

ISSN : 2272-9992

Éditeur

Société française des traducteurs

Édition imprimée

Date de publication : 1 décembre 2016

Pagination : 38-41

ISSN : 0395-773X

\section{Référence électronique}

Percy Balemans, «Traduire la mode : un art à part entière », Traduire [En ligne], 235 | 2016, mis en ligne le 01 décembre 2018, consulté le 21 septembre 2021. URL : http://journals.openedition.org/traduire/ 840 ; DOI : https://doi.org/10.4000/traduire.840 


\section{Traduire la mode : un art à part entière}

\section{Percy Balemans}

Traductrice indépendante (de l'anglais et de l'allemand vers le néerlandais) depuis 2007, ma toute première incursion dans le domaine de la mode a été la traduction d'articles de blog d'une marque de vêtements. À l'époque, mon expérience en la matière se limitait à un exposé en langue française sur l'histoire de la mode lorsque j'étais encore au lycée, bien des années auparavant. Passionnée très rapidement par le sujet, j'ai trouvé tout naturel d'en faire l'une de mes spécialisations.

Les textes que je traduis pour le secteur de la mode sont principalement des campagnes publicitaires et du contenu web pour des marques, ainsi que des articles pour un magazine de mode. L'un des projets les plus intéressants et les plus difficiles que j'ai eu à mener à bien a été la traduction, de l'anglais vers le néerlandais, de l'intégralité des textes de l'exposition " La planète mode Jean Paul Gaultier : de la rue aux étoiles " présentée au Kunsthal de Rotterdam en 2013(1). Créée par le Musée des beaux-arts de Montréal, l'exposition a voyagé dans le monde entier entre 2011 et 2016, faisant notamment escale à Paris, au Grand Palais, pendant l'été 2015(2).

Pour moi, l'un des aspects les plus satisfaisants de ce métier est de ne jamais savoir au préalable le type de texte que j'aurai à traduire le lendemain, et ce alors même que je suis spécialisée dans des domaines bien précis. Bien évidemment, je connaissais déjà Jean Paul Gaultier ; ce projet a toutefois été l'occasion idéale d'en apprendre davantage sur "l'enfant terrible de la mode " et sur son œuvre.

Les textes qui m'ont été confiés étaient en fait les panneaux d'information devant être affichés dans les différentes salles, ainsi que les descriptions de chacune des créations présentées. D'emblée, j'ai échangé avec la commissaire d'exposition sur le style et le ton à adopter, chaque client ayant à cet égard ses propres idées, en fonction du public visé. Comme il n'est pas

(1) NdT : Lors de son passage à Paris, l'exposition a été simplement rebaptisée " Jean Paul Gaultier ".

(2) https://www.jeanpaulgaultier.com/en-us/spectacles/jpgexpo/ 
toujours simple de s'en tenir à des considérations théoriques, j'ai pris l'habitude de soumettre à mes clients un échantillon de ma traduction, le plus en amont possible, en vue de trouver avec eux le style qui leur convient le mieux. Par ailleurs, je demande systématiquement d'autres documents susceptibles de m'être utiles : textes déjà rédigés ou traduits (le cas échéant) à titre de référence, glossaires éventuels, guides de style, etc.

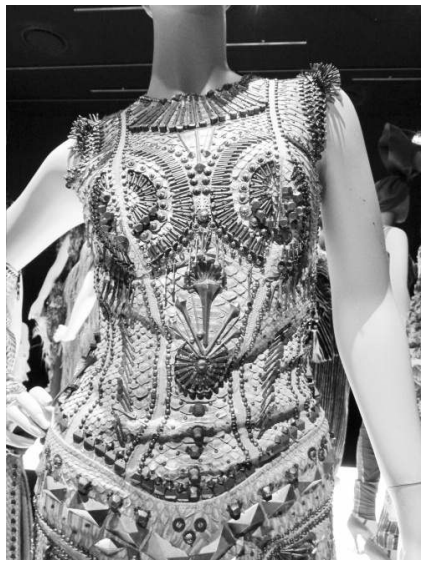

Broderie " barbare " à clous.

Le projet ayant commencé bien avant le vernissage de l'exposition, je me suis empressée de demander à la commissaire s'il existait des visuels des créations présentées, mais celle-ci n'avait à sa disposition que des images miniatures offrant très peu de détails. La traduction des descriptions s'est donc avérée particulièrement délicate : en effet, Jean Paul Gaultier est connu pour son utilisation de matières et de techniques inhabituelles, qu'il faut voir de ses propres yeux pour bien comprendre. Par exemple, dans la description en anglais d'une des robes figurait l'expression nail embroidery. N'étant pas spécialiste de la broderie, je me suis d'abord demandé s'il s'agissait d'une technique de réalisation particulière. Heureusement pour moi, la rétrospective avait déjà été présentée dans plusieurs pays et certaines personnes avaient mis en ligne des photos prises lors de leur visite. Ma petite enquête m'a donc finalement permis de trouver un gros plan de la robe... sur laquelle se trouvaient effectivement des clous soigneusement intégrés à la broderie !

Une autre description pour laquelle la recherche d'un visuel a été absolument primordiale : velvet cassock opening to reveal an icon. Le mystère a été dûment éclairci grâce à la photo où l'on voyait bien une soutane faite de velours, ornée de deux petites "portes" s'ouvrant pour révéler une icône religieuse. Ces deux exemples démontrent bien toute l'importance des visuels pour le traducteur.

J'ai également été confrontée à une autre difficulté du fait de la présence dans le texte anglais de quelques coquilles prêtant à confusion. Par exemple, wood pants (pantalon en bois) correspondait en réalité à wool pants (pantalon en laine). Dans d'autres conditions, j'aurais tout de suite relevé l'erreur, mais au point où j'en étais, je n'aurais pas été surprise de trouver dans les collections de Jean Paul Gaultier un pantalon en bois...

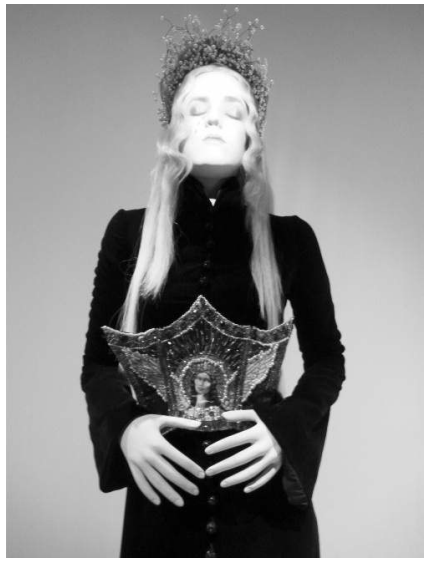

Soutane en velours avec ouverture " icône surprise ". 
La veille du vernissage, la commissaire m'a confié quelques phrases à traduire à la dernière minute, en l'occurrence la description de créations qui venaient d'être ajoutées à l'exposition.

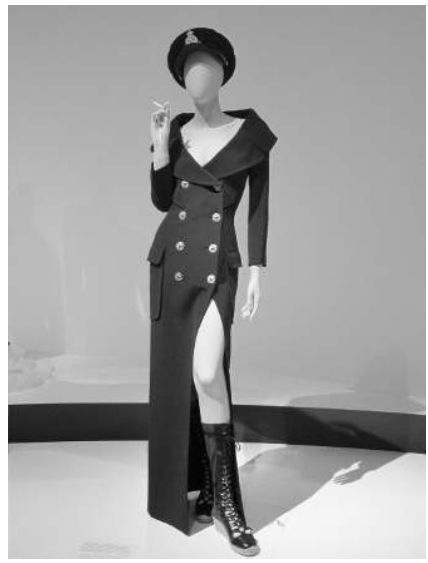

Robe caban à grand décolleté.

Pour l'une d'entre elles, l'opération s'est avérée particulièrement complexe, puisque le texte dont je disposais était French dress in silk: robe caban en saté (sic) à grand décolleté. Aucune image n'était fournie et la deuxième partie de la description était en français, langue qui ne figure pas dans mes combinaisons de travail. Les seules informations en ma possession étaient le nom, l'année et la saison de la collection dont la robe faisait partie, ainsi qu'un commentaire précisant qu'elle avait été portée par Catherine Deneuve.

À l'aide d'un dictionnaire français, j'ai réussi à comprendre qu'un caban était une veste portée par les matelots et qu'il s'agissait donc d'une robe inspirée d'un uniforme de marin : logique, puisque le thème de la marine a été une constante dans les créations de Jean Paul Gaultier. Comme je n'arrivais toujours pas à comprendre ce que signifiait " en saté ", j'ai fait appel à une consœur française. Selon elle, il s'agissait d'une coquille et il fallait plutôt comprendre " en satiné ", ce qui semblait tout à fait vraisemblable. J'ai fini par trouver une photo en recherchant dans la collection concernée une robe d'inspiration marine avec un "grand décolleté ". Quelques recherches sur Internet plus tard, j'ai eu la confirmation que Catherine Deneuve avait effectivement porté la robe en question. Mission impossible... accomplie ! J'ai envoyé la traduction à la commissaire d'exposition, en lui demandant de bien vouloir vérifier la description avec la robe sous les yeux, histoire d'en avoir le cœur net.

Dans un monde idéal, j'aurais préféré voir toutes les pièces de l'exposition avant son ouverture, afin de valider mes traductions. Or, cela s'est avéré impossible, les traductions devant être bouclées et mises sous presse bien en amont de l'arrivée des créations à Rotterdam. Fort heureusement, comme j'ai pu le constater en visitant l'exposition, mes traductions collaient parfaitement aux modèles.

J'ai particulièrement aimé travailler sur ce projet, qui m'a permis de beaucoup apprendre sur Jean Paul Gaultier et sur ses créations. D'ailleurs, quel plaisir de pouvoir les admirer lors du vernissage (broderie à clous incluse !). L'exposition m'a même laissé une si forte impression que je suis retournée la voir l'année suivante lors de son passage à Londres. 
Texte traduit de l'anglais par Nelia Fahloun.

Diplômée de l'école de traduction et d'interprétation des Pays-Bas en 1989, Percy Balemans a d'abord été traductrice salariée en agence pendant quelques années, avant de rejoindre une entreprise d'informatique, où elle a été successivement rédactrice technique, chargée de conception, rédactrice web et formatrice. Traductrice indépendante à temps plein depuis 2007, Percy traduit depuis l'anglais et l'allemand vers le néerlandais dans les domaines de la publicité (transcréation), de la mode, de l'art, des voyages et du tourisme. Elle est en outre membre de plusieurs associations professionnelles : l'association néerlandaise des traducteurs et interprètes (NGTV), l'American Translators Association (ATA), ainsi que le Chartered Institute of Linguists (CIOL), en tant que Chartered Linguist (Translator).

Photos prises par Percy Balemans au Kunsthal de Rotterdam. 